\title{
Origami-inspired Shape Memory Folding Microactuator
}

\author{
Lena Seigner 1, Olha Bezsmertna 2, Sebastian Fähler ${ }^{2}$, Georgino Tshikwand ${ }^{3}$, Frank Wendler ${ }^{3}$ \\ and Manfred Kohl 1,* \\ 1 Ins titute of Microstructure Technology, Karlsruhe Institute of Technology, 76344 Eggenstein- \\ Leopoldshafen, Germany \\ 2 Leibniz IFW Dresden, Helmholtzstr. 20, 01069 Dresden, Germany \\ 3 Institute of Materials Simulation, Frie drich-Alexander University of Erlangen-Nürnberg (FAU), 90762 \\ Fürth, Germany \\ * Correspondence: manfred.kohl@kit.edu
}

\begin{abstract}
This paper presents the design, fabrication and performance of origami-based folding microactuators based on NiTi films showing the one-w ay shape memory effect. Freestanding NiTi films are micromachined by laser cutting or photolithography to achieve double-beam structures allowing for direct Joule heating with an electrical current. The NiTi microactuators are interconnected to rigid sections (tiles) forming an initial planar system that self-folds into a predetermined 3D shape upon heating. A thermo-mechanical treatment is used for shape setting of as-received specimens to approach a maximum folding angle of $180^{\circ}$. The bending moments, bending radii and load-dependent folding angles upon Joule heating are evaluated. The shape setting process is particularly effective for small bending radii, which, how ever generates residual plastic strain. After shape setting, unloaded beam structures show recoverable bending deflection betw een $0^{\circ}$ and $140^{\circ}$ for a maximum heating pow er of $900 \mathrm{~mW}$. By introducing additional loads to account for the effect of the tiles, the smooth folding characteristic evolves into a sharp transition, whereby full deflection up to $180^{\circ}$ is reached.
\end{abstract}

Keywords: self-folding origami; microactuation; shape memory alloy films; microtechnology

\section{Introduction}

Origami is the widely known art of paper folding, by which flat sheets of paper are transformed into three-dimensional shapes. These ancient techniques provide the inspiration for promising future applications in science and engineering, e.g. assembly and robotics. Particular advantages are for example the compact setup of deployable structures (e.g. airbags [1, 2], wings [3] or biomedical devices), as well as a reduced complexity of fabrication process where2D structures can be assigned their actual function after the fabrication.

The use of smart materials can enable folding under the influence of a non-mechanical field. Attractive candidates are shape memory alloys (SMAs) that exhibit high bending moments, low corrosion and low fatigue. The special feature of SMAs is their thermally activated solid-state phase transition. The crystal realignment from the low temperature phase, called martensite, to the high temperature phase (austenite) is diffusionsless and reversible. If the SMA film used in this work is deformed in its martensite state, it can be restored to its memory form by heating above the phase transition temperature (austenite finish temperature Af) by using the one-w ay shape memory effect.

Previous concepts for origami-inspired self-folding SMA sheets have been published in [4] and [5]. Further relevant research has been performed, e.g., in the field of robogami [6-8]. Alternative mechanisms for satellite deployment mechanisms using SMA hinges were presented in [9]. While 
previous developments focused on the macro scale, we intend to transfer the concept of origamiinspired SMA folding actuation to the micro scale by combining state-of-the art methods of SMA film engineering and micromachining. The aim of this study is to design, fabricate and analyze the performance of the SMA folding microactuators. For this we present first results on the grow th of 1 $\mu \mathrm{m}$ thin films and detailed experiments on $20 \mu \mathrm{m}$ thick films, where we demonstrate shape setting and bending actuation.

\section{Materials and Fabrication}

Here we use NiTi films as active material which serve as interconnecting hinges betw een single triangular tiles. The material undergoes a tw o step solid-to-solid phase transformation while cooling down. Instead of directly transforming into martensite, it undergoes an intermediate R-phase transformation. The relevant phase transformation temperatures of the NiTi material (depicted in Table 1) are obtained by differential scanning calorimetry and temperature dependent electrical resistance measurements. At room temperature the SMA material is in R-phase condition. By folding, the stress-induced martensitic transformation is initiated and by heating above $62^{\circ} \mathrm{C}$ the reoriented martensite transforms into austenite. Cooling back to room temperature in stress-free state leads to the formation of R-phase again. Finite element (FE) simulations are performed to describe the thermo-mechanical performance of NiTi test devices. In order to evaluate the selected material model (Lagoudas model [10]) describing the SMA material behavior, tensile test simulations w ere conducted. Material parameters from our tensile experiments shown in Table $\mathbf{1}$ were provided as input. Figure 1 show s both, the experimental and simulation data of the stress-strain behavior. It can be stated that the simulation allows to approximate the macroscopic material behavior under tensile loading. It shows a higher critical stress for the intermartensitic transformation by around $25 \%$ and underestimates the slope of the stress-plateau. By assuming an ideal material, the simulations cannot accurately represent the actual transformation slopes obtained from experiments. We attricute this to microscopic material imperfections such as internal defects introduced by the fabrication process.

Table 1. Simulation Parameters

\begin{tabular}{rcc} 
Parameter & Abb. & Value \\
\hline Martensite Start & $\mathrm{M}_{\mathrm{s}}$ & $19^{\circ} \mathrm{C}$ \\
Martensite Finish & $\mathrm{M}_{\mathrm{f}}$ & $9^{\circ} \mathrm{C}$ \\
Austenite Start & $\mathrm{As}_{\mathrm{s}}$ & $52^{\circ} \mathrm{C}$ \\
Austenite Finish & $\mathrm{Af}_{\mathrm{f}}$ & $62^{\circ} \mathrm{C}$ \\
R-phase peak & $\mathrm{R}_{\mathrm{p}}$ & $45^{\circ} \mathrm{C}$ \\
Young's Modulus & $\mathrm{EM}_{\mathrm{M}}$ & $25 \mathrm{GPa}$ \\
Clausius-Clapeyron & $\mathrm{C}$ & $8.1 \mathrm{MPa} / \mathrm{K}$ \\
TransformationStrain & $\varepsilon^{t}$ & 0.035 \\
\hline
\end{tabular}

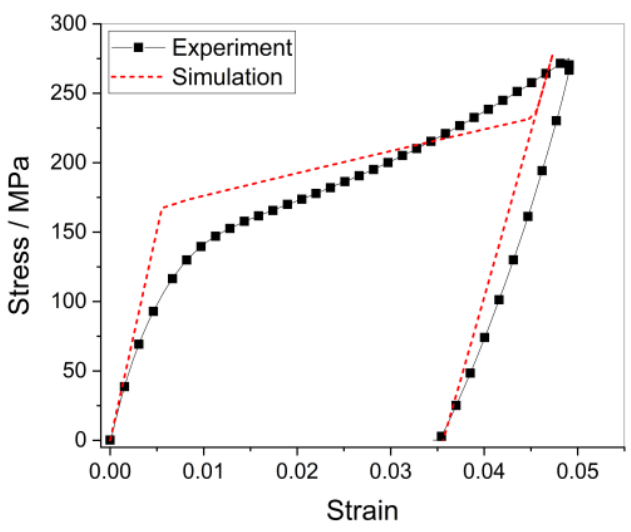

Figure 1. FEM simulation and comparison with experimental tensile testresults

The intended fabrication process can be divided into four main steps (see Figure 2). The first two steps comprise magnetron sputtering of NiTi films onto a silicon substrate as well as the sacrificial layer technology to release the SMA films from the substrate. The minitaturization of the folding actuators requires thin film technology. Therefore, the increase of martensite to austenite transformation temperature by addition of $\mathrm{Cu}$ is investigated, motivated by [11]. The transformation is shifted tow ards higher temperatures (see Figure 3). The finish temperature of direct transformation $(\mathrm{Mf})$ increased from $-126^{\circ} \mathrm{C}$ to $-85^{\circ} \mathrm{C}$, the corresponding temperature of the reverse transformation to austenite $\left(\mathrm{As}_{\mathrm{s}}\right)$ increased from $-121^{\circ} \mathrm{C}$ to $-72^{\circ} \mathrm{C}$. Steps 3 and 4 include film structuring, shape setting and bonding to tiles. So far, we used freestanding NiTi films of $20 \mu \mathrm{m}$ thickness as the currently sputtered films still have transformation temperatures below room temperature. The SMA microactuator s are micromachined by laser cutting which has the advantage of cost-effectiveness and 
speed. Alternatively, we investigated micro structuring by photolithography which will prove to be more precise with further downscaling of the design. The chosen design of a double beam structure allow s to apply an electric current across the two ends to actuate the SMA microactuators by direct

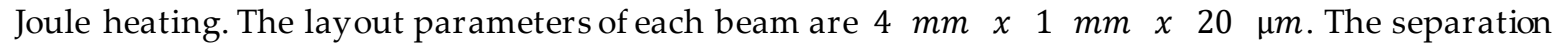
between the two beams is $4 \mathrm{~mm}$. The memory shape is the folded state at $180^{\circ}$ and is imprinted by an annealing process in a vacuum furnace at $460^{\circ} \mathrm{C}$ for $30 \mathrm{~min}$. In order to set the memory shape the specimens are placed and folded by $180^{\circ}$ in an annealing jig and covered by thin Ti foils to avoid surface oxidation during the shape setting process. Two different folding radii are realized (0.5 and $1 \mathrm{~mm}$ ). The structure is then glued between two Kapton tiles and electrically contacted by gap welding.

(1) Sputter deposition of $\mathrm{NiTi}$

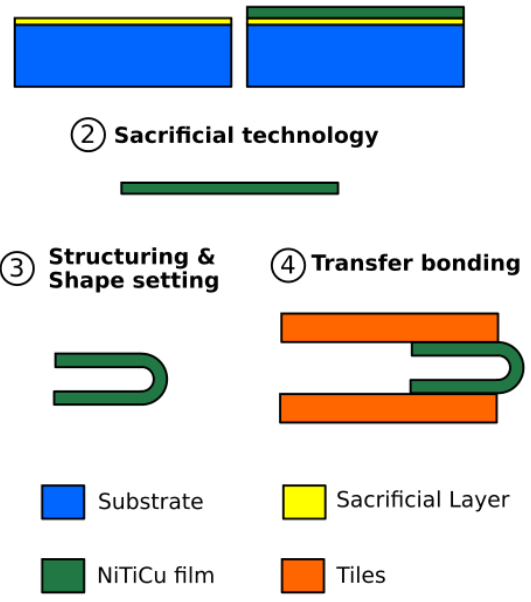

Figure 2. Process flow for fabrication of SMA folding microactuators

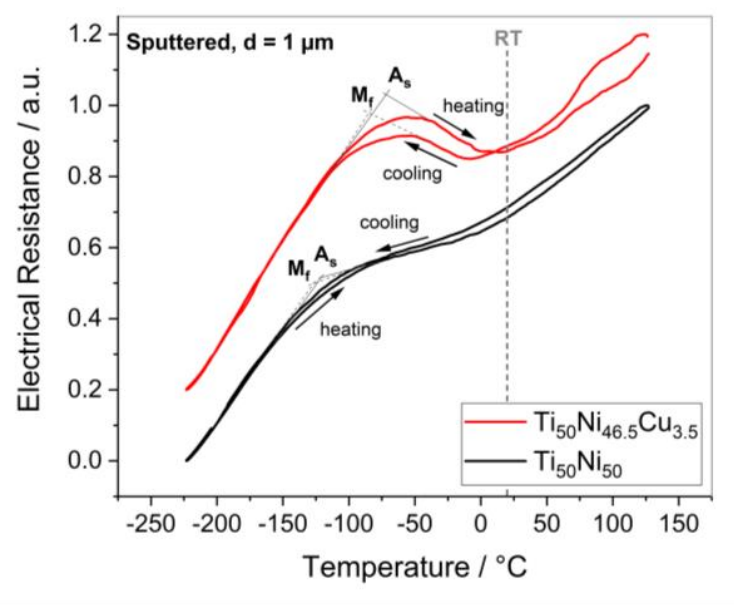

Figure 3. Temperature de pendent electrical resistance measurements of sputtered NiTi (annealed at $650^{\circ} \mathrm{C}$ for $10 \mathrm{~min}$ ) and $\mathrm{NiTiCu}$ (annealed at $500^{\circ} \mathrm{C}$ for $30 \mathrm{~min}$ ) films.

\section{Results}

Figure 4 shows the active folding of a SMA microactuator consisting of a $20 \mu \mathrm{m}$ thick NiTi double-beam cantilever after the shape setting process. The microactuator has been mechanically unfolded at room temperature and subsequently heated above austenite finish temperature. The microscope images show that theSMA hinge almost completely recovers its memory shape.

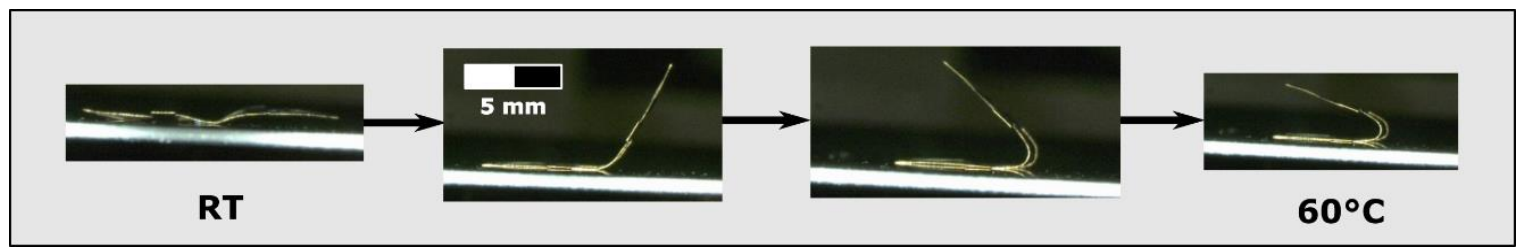

Figure 4. Folding sequence of a SMA microactua tor that has been shape set to $180^{\circ} \mathrm{C}$ after me chanical unfolding at room temperature (RT). Recovery of the memory shape is obtained by uniform heating above the austenite finish temperature Af.

In the following, we present results on two different Origami devices consisting of a SMA microactuator and two tiles of either Kapton or ceramic. Investigations include IR thermography and camera tracking of the bending angle during direct Joule heating of the microactuator. Figure 5 shows two characteristics of folding angles as a function of applied voltage for Joule heating, which correlates with the temperature of the microactuator. The folding radius of the specimen is $0.5 \mathrm{~mm}$. Here, the state "no load" refers to the pure microactuator without tiles. When the folding axis of the Origami device is aligned perpendicular to the gravitational field (see Figure 5(a)) the starting voltage 
for folding is shifted to higher voltages (from around $1.8 \mathrm{~V}$ to $2.4 \mathrm{~V}$ ) and the characteristic shows a steeper behavior compared to the case where the folding axis is aligned along the gravitational field. This behavior can be attributed to stress-induced increase of SMA transformation temperatures. A similar trend can be observed by adding additional weight as shown in Figure $\mathbf{5}(\mathbf{b})$. In unloaded case the microactuator folds back to around $65^{\circ}$ and while cooling down it reopens slightly. By adding the load of the Kapton tile, the folding angle remains constant at room temperature. In the case of a ceramic tile causing a gravitational load of $3.8 \mathrm{mN}$, the microactuator folds down to $10^{\circ}$ upon heating and by subsequent cooling to room temperature it reaches the maximum total folding angle of $180^{\circ}$.

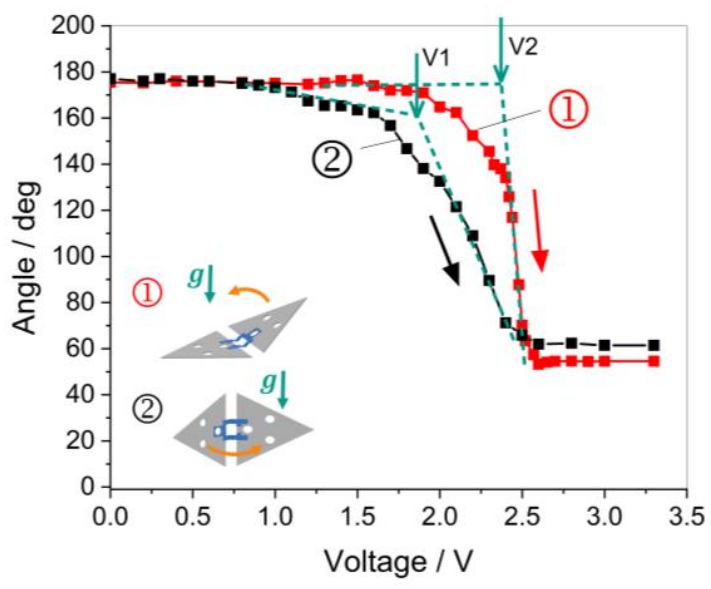

(a)

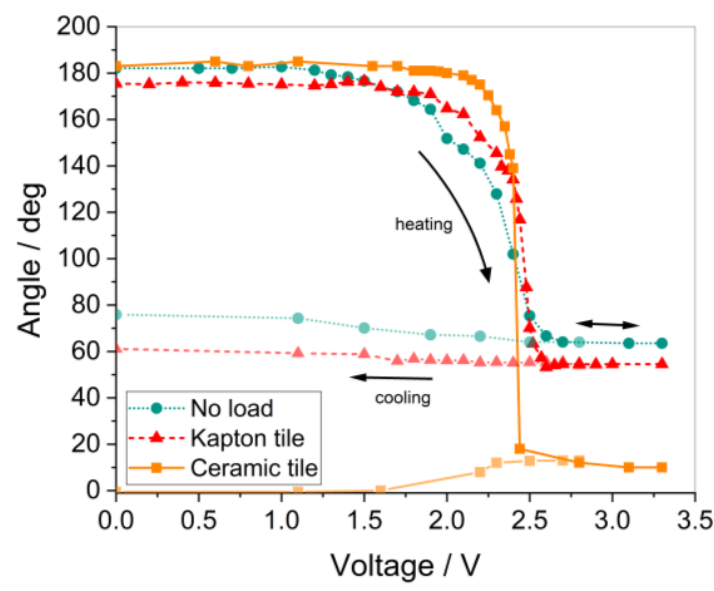

(b)

Figure 5. Folding angle of Origami devices as a function of applied voltage for Joule heating determined by ca mera tracking: (a) Devices with Kapton tiles a re tested in two diffe rent orientations of folding axis along and perpendicular to the gravitational field as illustrated in the inset; (b) Influence of gravitational load of the tile material on the folding angle upon heating and cooling.

These measurements show that the performance of Origami devices is tunable by suitable loading. How ever, at small load, the microactuator only folds back to $60^{\circ}$. This incomplete shape recovery is a consequence of residual plastic deformation. Samples with an increased folding radius of $1 \mathrm{~mm}$ show less residual strain but the shape setting process is less effective and samples with higher bending radii show larger opening angles after heat treatment.

Additionally we investigate the torque generated by the restoring movement of the unfolded SMA microactuator back to its memory shape. Here, theSMA microactuator is unfolded by $180^{\circ}$ and positioned below a load cell with one end fixed on a platform (see Figure 6(a)). Upon Joule heating, shape recovery of the microactuator is blocked and a restoring force acts on the load cell. The resulting torque is subsequently calculated by taking into account the distance to the fixation. Figure 6(b) shows the heating and cooling curves of the torque generated by the microactuator. A maximum torque of $0.047 \mathrm{Nmm}$ is reached at a voltage of $2.8 \mathrm{~V}$ which corresponds to a heating power of $900 \mathrm{~mW}$. When cooling down we can observe a hysteresis due to the difference in forw ard and backw ard phase transformation temperatures of the SMA. The remaining torque of $0.01 \mathrm{Nmm}$ corresponds to the torque required to unfold the actuator to $180^{\circ}$ which is four times smaller than the folding torque generated upon heating. Thus, by combining two counteracting antagonistic microactuators reversible folding in the range of $\pm 180^{\circ}$ is feasible. 


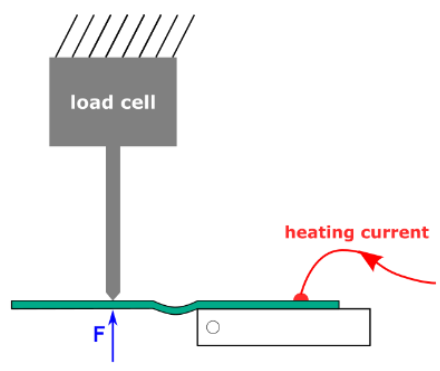

(a)

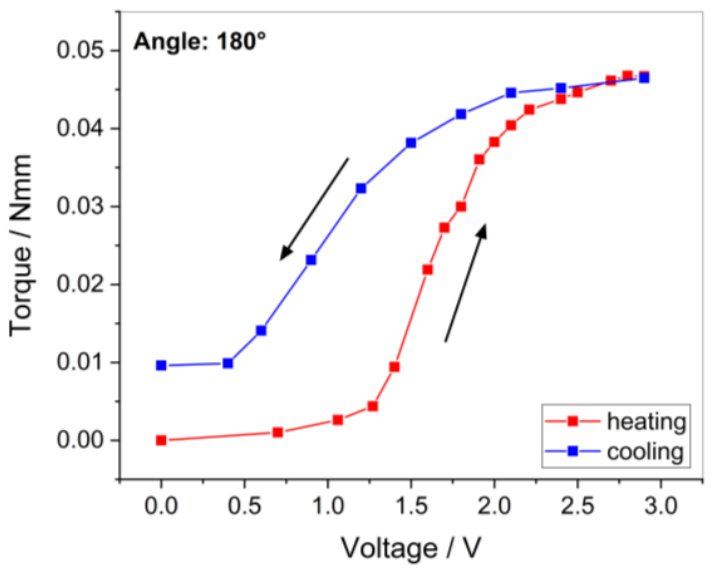

(b)

Figure 6. Torque measurement of a SMA microactuator upon electrical heating that has been shape set to $0^{\circ}$ and subsequently unfolded to $180^{\circ}$ (a) Measurement setup with a load cell, the torque is calculated by the measured force times the distance to the fixation; (b) heating and cooling characteristics

\section{Conclusions}

We present the design, fabrication and characterization of a shape memory microactuator for active folding based on the concept of origami. Double-beam cantilever structures are designed and shape-set for $180^{\circ}$ folding using Joule heating. Different loading conditions have been investigated that allow for the tuning of the actuator system in terms of actuation time, heating power and folding angles. The maximum folding torque of $0.047 \mathrm{Nmm}$ should be sufficient to actuate an Origami device with antagonistic SMA microactuators for reversible folding. Further improvement of folding performance is expected by design optimization and improvements in the shape setting process. Ongoing investigations include Origami devices with decreasing film thickness and tailored phase transformation temperatures.

Acknowledgments: This research has received funding from the German Research Foundation (DFG) within the priority programme "SPP2206 - Cooperative Multistage Multistable Microactuator Systems", projects KO 2953/10-1, FA 453/15-1 and WE4747/5-1.

Author Contributions: M.K., S.F. and F.W. developed the concept; L.S. designed and performed the experiments; analyzed the data and wrote the draft of the manuscript; O.B. performed sputtering at IFW Dresden; G.T. provided material simulation models; M.K. supervised the work, reviewed and edited the manuscript.

Conflicts of Interest: The authors declare no conflict of interest

\section{Abbreviations}

IR: Infrared

SMA: Shape Memory Alloy

\section{References}

1. Cromvik, C. and K. Eriksson, Airbag folding based on origa mi mathematics, in Fourth International Meeting of Origami Science, Mathematics, and Education. 2006.

2. Hoffman, R., Airbag folding: origami design applied to an engineering problem, in Third International Meeting of Origami Science, Mathematics and Education. 2001: Asilomar, CA. 
3. Baek, S.-M., D.-Y. Lee, and K.-J. Cho. Curved Compliant Facet Origami-Ba sed Self-Deployable Gliding Wing Module for Jump-Gliding. in ASME 2016 International Design Engineering Technical Conferences and Computers and Information in Engineering Conference. 2016.

4. Hawkes, E., et al., Programmable matter by folding. Proceedings of the National Academy of Sciences, 2010. 107(28).

5. Peraza-Hernandez, E.A., D.J. Hartl, and R.J. Malak, Design and numerical analysis of an SMA mesh-based self-folding sheet. Smart Materials and Structures, 2013. 22(9).

6. Firouzeh, A., et al., Sensor and actuator integrated low-profile robotic origami, in IEEE/RSJ International Conference on Intelligent Robotsand Systems. 2013: Tokyo. p. 4937-4944.

7. Paik, J.K. and R.J. Wood, A bidirectional shape memory alloy folding actuator. Smart Materials and Structures, 2012. 21(6): p. 065013.

8. Zhakypov, Z., J.-L. Huang, and J. Paik, A Novel Torsional Shape Memory Alloy Actuator: Modeling, Characterization, and Control. IEEE Robotics E Automation Magazine, 2016. 23: p. 65-74.

9. Jeong, J.W., etal., A novel tape spring hinge mechanism for qua si-static deployment of a satellite de ployable using shape memory alloy. Review of Scientific Instruments, 2014. 85.

10. Lagoudas, D.C., Shape Memory Alloys. 2008: Springer.

11. Ishida, A., M. Sato, and Z.Y. Gao, Properties and applications of TiNiCu shape-memory-alloy thin films. Journal of Alloys and Compounds, 2013. 577.

(C) 2020 by the authors; licensee MDPI, Basel, Switzerland. This article is an open access article dis tribute $\mathrm{d}$ under the terms and conditions of the Creative Commons by Attribution (CC-BY) license (http://creativecommons.org/licenses/by/4.0/). 